\title{
About the attractor-repeller points during the descent of an asymmetric spacecraft in the atmosphere
}

\author{
V.V. Lyubimov ${ }^{1}$, V.S. Lashin ${ }^{1}$

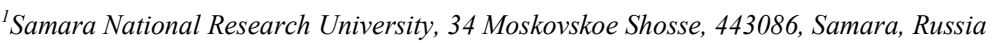

\begin{abstract}
The aim of this study is to analyze the resonant attractor-repeller points during the atmospheric descent of a spacecraft with small asymmetry. The mathematical simulation of spacecraft rotational motion uses an approximate non-linear system of equations obtained by the method of integral manifolds. Application of the averaging method and the Lyapunov method makes it possible to obtain realization conditions of attractor-repeller points on non-resonance parts of the motion. By analyzing of the said conditions, we have identified specific cases when the principal resonance is either an attractor point or a repeller point.
\end{abstract}

Keywords: resonance; attractor; repeller; averaging; spacecraft; atmosphere; asymmetry

\section{Introduction}

Various resonance phenomena in the problem of uncontrolled descent of a spacecraft with a small asymmetry in the atmosphere are explored in [1-2, etc.]. In particular, the disturbing moments of mass-aerodynamic asymmetry can leads to the evolution of angular velocity of the spacecraft to the resonance values [3-5]. In this case, the non-resonance evolution of the angular velocity of the asymmetric spacecraft is the secondary resonance effect [6]. The external stability of resonance is considered in the problems of perturbed rotational motion of the asymmetric spacecraft in the atmosphere or satellite in orbit $[5,7]$. The realization of the main resonance leads to significant increase of the angle of attack. In practice, this can lead to emergency situation during the deployment of the parachute system of a spacecraft. It is known that the external stability of the resonance contributes to the evolution of the variables of the dynamic system to resonant values (resonant attractor). Therefore, the study of the phenomenon of external stability of resonance is an important practical task. This phenomenon arises when the condition of the external stability of the resonance is satisfied. It should be noted that the investigation of the resonant attractor and repeller is supposed to be performed in a more general form in comparison with the results presented in [5].

\section{Problem statement}

Let us assume that spacecraft is a solid body in the form of a cone combined with a spherical surface. Let the axis $O X$ is the symmetry axis of the cone. In the process of entry into atmosphere, a spacecraft is directed its conical surface along of the air flow. During the atmospheric descent, the spacecraft engages in the precessional motion. It is known that a spacecraft receives small angular momentum when undocked from the base orbital module [8]. In this case, the angular momentum lead to the formation of the components of the angular velocity of the spacecraft $\omega_{x}(0), \omega_{y}(0), \omega_{z}(0)$. These components of the angular velocity are recorded of the spacecraft body-fixed coordinate system OXYZ. Suppose that these angular velocities are initial when the spacecraft enters into the atmosphere. The origin of the coordinate system $O$ is located at the center of mass of the spacecraft. In [9] it is shown that the resonance values of the angular velocity $\omega_{\mathrm{x}}$ can be determined on the basis of the method of integral manifolds [10]. The values of angular velocity $\omega_{\mathrm{x}}$ corresponding to the principal resonance are defined as follows [5]: $\omega_{x}^{r}= \pm \omega /\left(1-I_{x}\right)^{1 / 2}$. Here $\omega=\left(-m_{z n} q S L \operatorname{ctg} \alpha / I\right)^{1 / 2}$ is the angular velocity, $m_{\mathrm{zn}}$ is the restoring moment coefficient for the angle of attack $\alpha, q$ is the dynamic pressure, $S$ is the area of the maximum cross section of a spacecraft, $L$ is the length of a spacecraft, $\bar{I}_{x}=I_{x} / I, I=\left(I_{\mathrm{y}}+I_{\mathrm{z}}\right) / 2, I_{\mathrm{x}}, I_{\mathrm{y}}, I_{\mathrm{z}}$ are the principal moments of inertia of a spacecraft. It is known that the principal resonance has the greatest influence on the evolution of slow variables on the non-resonant parts of the motion, compared with resonances of higher orders. The aim is to study the realization conditions of resonant attractor and resonant repeller in case of atmospheric descent of a spacecraft with small aerodynamic-inertial asymmetry. Let the attack angle take arbitrary values. We apply the method of averaging and the Lyapunov method to the research of attractors and repellers.

\section{Methods}

\subsection{Mathematical model}

The approximate non-linear system of equations of motion of a spacecraft with small aerodynamic-inertial asymmetry, describing the motion of a spacecraft relative to the center of mass has the form [5]:

$$
\overline{\mathrm{I}}_{\mathrm{x}} \frac{\mathrm{d} \omega_{\mathrm{x}}}{\mathrm{dt}}=\varepsilon m^{\Delta} \omega_{1,2}^{2} \operatorname{tg}{ }^{2} \alpha \cos \left(2 \theta+2 \theta_{3}\right)
$$




$$
\begin{aligned}
& \frac{F_{a}}{4 \omega_{a}^{2}} \frac{d \alpha}{d t}=-\varepsilon \frac{\omega \operatorname{tg} \alpha}{2 \omega_{a}^{2}} \frac{d \omega}{d t} \quad \varepsilon \frac{m^{A}}{2 \omega_{a}} \cos \left(\theta+\theta_{1}\right)- \\
& -\varepsilon \frac{\omega_{1,2} \operatorname{tg} \alpha}{4 \omega_{a}^{2}}\left[\left(10+\overline{\mathrm{I}}_{\mathrm{x}}\right) \omega_{x} \omega_{1,2}-2\left(2+\overline{\mathrm{I}}_{\mathrm{x}}\right) \omega_{x}^{2}\right] m^{\Delta} \cos \left(2 \theta+2 \theta_{3}\right)- \\
& -\varepsilon \frac{\omega_{1,2} \operatorname{tg} \alpha}{4 \omega_{a}^{2}}\left[\left(\operatorname{tg}^{2} \alpha-4\right) \omega_{1,2}^{2}\right] m^{\Delta} \cos \left(2 \theta+2 \theta_{3}\right), \\
& \frac{\mathrm{d} \theta}{\mathrm{dt}}=\omega_{\mathrm{x}}-\omega_{1,2}, \\
& \frac{\mathrm{d} \omega}{\mathrm{dt}}=\varepsilon \frac{\omega}{2 \mathrm{q}} \frac{\mathrm{dq}}{\mathrm{dt}} .
\end{aligned}
$$

Here $\varepsilon$ is the small parameter, $\theta=\varphi-\pi / 2, \varphi$ is aerodynamic roll angle; $m^{A}, m^{\Delta}, \theta_{1}, \theta_{3}$ are functions that characterize the values and relative positions of the aerodynamic and inertial asymmetries of the spacecraft,

$m^{A}=\sqrt{\left(m_{1}^{A}\right)^{2}+\left(m_{2}^{A}\right)^{2}}, m_{1}^{A}=-\frac{\left(1+\bar{I}_{x}\right) \omega_{x}-3 \omega_{1,2}}{2 \omega_{a}} \frac{\omega^{2}}{m_{z n}}\left(m_{y}^{f}-C_{x} \overline{\Delta z}\right) \operatorname{tg} \alpha-\frac{\omega_{1,2} \omega^{2} \operatorname{tg}^{2} \alpha}{2 \omega_{a} m_{z n}}\left(C_{y n} \overline{\Delta z}\right)$

$\frac{\bar{I}_{x z}}{2 \omega_{a}}\left[\omega_{x} \omega_{1,2}\left(\omega_{x}+\omega_{1,2} \operatorname{tg}^{2} \alpha\right)-\omega_{x}^{2}\left(\omega_{x} 2 \omega_{a}\right)\right], m_{2}^{A}=-\frac{\left(1+\bar{I}_{x}\right) \omega_{x}-3 \omega_{1,2}}{2 \omega_{a}} \frac{\omega^{2}}{m_{z n}}\left(m_{z}^{f}+C_{x} \overline{\Delta y}\right) \operatorname{tg} \alpha+$

$+\frac{\omega_{1,2} \omega^{2} \operatorname{tg}^{2} \alpha}{2 \omega_{a} m_{z n}}\left(C_{y n} \overline{\Delta y}\right) \pm \frac{\bar{I}_{x y}}{2 \omega_{a}}\left[\omega_{x} \omega_{1,2}\left(\omega_{x}+\omega_{1,2} \operatorname{tg}^{2} \alpha\right)-\omega_{x}^{2}\left(\omega_{x} 2 \omega_{a}\right)\right], \sin \theta_{1}=m_{1}^{A} / m^{A}, \cos \theta_{1}=-m_{2}^{A} / m^{A}, \omega_{a}=\sqrt{\bar{I}_{x}^{2} \omega_{x}^{2} / 4+\omega^{2}} ;$ $m^{\Delta}=\sqrt{\bar{I}_{y z}^{2}+\overline{\Delta I}^{2}}, \sin 2 \theta_{3}=\overline{\Delta I} / m^{\Delta}, \cos 2 \theta_{3}=-\bar{I}_{y z} / m^{\Delta}, \bar{I}_{x y}=I_{x y} / I, \bar{I}_{x z}=I_{x z} / I, \bar{I}_{y z}=I_{y z} / I, \overline{\Delta I}=\Delta I / I$ are dimensionless moments of inertia of a SC, $\omega_{1,2}=\frac{\bar{I}_{x} \omega_{x}}{2} \pm \omega_{a} ; \omega_{x}-\omega_{1,2}$ is the resonant ratio of frequencies; $C_{x}, C_{y n}$ are the aerodynamic coefficients; $m_{y}^{f}, m_{z}^{f}$ are the coefficients of small moments caused by asymmetric shape of the spacecraft; $\overline{\Delta y}=\Delta y / L$, $\overline{\Delta z}=\Delta z / L ; \Delta y, \Delta z$ are small displacements of the center of mass of the spacecraft; $F_{a}=F_{a}\left(\omega_{x}, \alpha, \omega\right)$ is the known function of slow variables [5]. In equations (1)-(4) we consider the principal resonance, corresponding to the following condition: $\Delta=\omega_{x}-\omega_{1,2} \cong 0$. There are signs " \pm " and “ " in the equations (1)-(3). We assume in the said equations that the upper sign is selected when $\omega_{\mathrm{x}}>0$, and the lower sign is selected when $\omega_{\mathrm{x}}<0$. In the numerical simulation of spacecraft motion, the system of equations (1)-(4) should be considered together with the system of three differential equations for slowly varying of the center of mass parameters: the local flight-pass inclination angle $\vartheta(t)$, the spacecraft airspeed $V(t)$ and the spacecraft altitude $H(t)$ [1].

\subsection{Averaging and analysis of resonant attractor}

After using the method of averaging on non-resonant parts of a spacecraft motion we obtain [5]:

$$
\begin{aligned}
& \left\langle\frac{d \omega_{x}}{d t}\right\rangle=\varepsilon^{3}\left\{\frac{\bar{m}^{A} g_{2} g_{3}}{\Delta^{3}} \frac{\partial}{\partial \alpha}\left(\bar{m}^{A} g_{3} \frac{\partial \Delta}{\partial \alpha}\right)-\frac{\bar{m}^{A} g_{3}}{\Delta^{2}} \frac{\partial}{\partial \alpha}\left(\bar{m}^{A} g_{3} \frac{\partial g_{2}}{\partial \alpha}\right)+\right. \\
& \left.+\frac{3\left(\bar{m}^{A} g_{3}\right)^{2}}{\Delta^{4}}\left(\Delta \frac{\partial \Delta}{\partial \alpha} \frac{\partial g_{2}}{\partial \alpha}-g_{2}\left(\frac{\partial \Delta}{\partial \alpha}\right)^{2}\right)\right\} \frac{m^{\Delta} \cos \left(2 \theta_{1}-2 \theta_{3}\right)}{8}, \\
& \left\langle\frac{d \alpha}{d t}\right\rangle=\varepsilon^{3}\left\{\frac{\bar{m}^{A} g_{3} g_{1}}{\Delta^{3}}\left[\frac{\partial}{\partial \alpha}\left(\bar{m}^{A} g_{3}\right) \frac{\partial \Delta}{\partial \alpha}-\bar{m}^{A} g_{3} \frac{\partial^{2} \Delta}{\partial \alpha^{2}}\right]-\frac{\bar{m}^{A} g_{3}}{\Delta^{3}} \frac{\partial}{\partial \alpha}\left(\bar{m}^{A} g_{3} \Delta\right) \frac{\partial g_{1}}{\partial \alpha}+\frac{g_{1}}{\Delta^{2}}\left[\frac{\partial}{\partial \alpha}\left(\bar{m}^{A} g_{3}\right)\right]^{2}-\right. \\
& -\frac{\bar{m}^{A} g_{2}}{\Delta^{3}} \frac{\partial g_{3}}{\partial \omega_{x}}\left[\frac{\partial}{\partial \alpha}\left(\bar{m}^{A} g_{3}\right) \Delta+2 \bar{m}^{A} g_{3} \frac{\partial \Delta}{\partial \alpha}\right]-\frac{\left(\bar{m}^{A}\right)^{2} g_{3}}{\Delta^{2}} \frac{\partial g_{1}}{\partial \alpha} \frac{\partial g_{3}}{\partial \alpha}+\frac{\left(\bar{m}^{A}\right)^{2} g_{3}^{2}}{2 \Delta^{4}} \frac{\partial \Delta}{\partial \omega_{x}}\left[7 g_{2} \frac{\partial \Delta}{\partial \alpha}-4 \Delta \frac{\partial g_{2}}{\partial \alpha}\right]+ \\
& \left.+\frac{\left(\bar{m}^{A}\right)^{2} g_{3}^{2}}{2 \Delta^{4}}\left[2 \Delta^{2} \frac{\partial^{2} g_{1}}{\partial \alpha^{2}}-g_{1}\left(\frac{\partial \Delta}{\partial \alpha}\right)^{2}\right]\right\} \frac{m^{\Delta} \cos \left(2 \theta_{1}-2 \theta_{3}\right)}{8}+\varepsilon^{3} g_{4} .
\end{aligned}
$$

Here $g_{1}=\frac{2 \omega_{a} \omega_{1,2} \sin \alpha}{F_{a}}\left(\omega_{x}+\frac{\omega_{1,2}^{2} \sin ^{2} \alpha}{2 \omega_{a}}\right), g_{2}=\frac{\omega_{1,2}^{2} \sin ^{2} \alpha}{\bar{I}_{x}}, g_{3}=\frac{2 \omega_{a} \omega^{2}}{F_{a}}, g_{4}=\frac{4 m_{z n} S L \omega_{a}^{2}}{I F_{a}} \frac{d q}{d t}$. 
Equations (4) and (5) describe the non-resonant evolution of the angular velocity $\omega_{x}$ and the angle of attack $\alpha$ caused by the effect of the principal resonance $\Delta=0$. At the positive values of $\omega_{x}$ resonant ratio is equal to $\Delta=\left(1-\frac{\bar{I}_{x}}{2}\right) \omega_{x}-\omega_{a}$. Let us assume that the spacecraft has the following ratio of the moments of inertia: $\bar{I}_{x}=2$. Here the resonant ratio is equal to

$$
\Delta=-\sqrt{\omega_{x}^{2}+\omega^{2}}
$$

We introduce the function $V\left(\omega_{x}, \omega\right)=\Delta^{2}$. This Lyapunov function can be written as:

$$
V\left(\omega_{x}, \omega\right)=\omega_{x}^{2}+\omega^{2}
$$

Here $\omega_{x}, \omega$ are determined from equations (5) and (4) respectively. Given the expression of (7), we see that the principal resonance $\Delta=0$ is realized at

$$
\left\{\begin{array}{c}
\omega_{x}=0 \\
\omega=0
\end{array}\right.
$$

Thus, the condition of the external stability of the principal resonance [5] has the following form:

$$
\frac{d V}{d t}=2 \omega_{x}\left\langle\frac{d \omega_{x}}{d t}\right\rangle+2 \omega \frac{d \omega}{d t}<0
$$

The condition (10) is a condition of asymptotic stability of a trivial solution (9). The fulfillment of the condition (10) provides for realization of a resonant attractor (9). On the contrary, the condition of external instability of the principal resonance is the following:

$$
\frac{d V}{d t}=2 \omega_{x}\left\langle\frac{d \omega_{x}}{d t}\right\rangle+2 \omega \frac{d \omega}{d t}>0
$$

The condition (11) is the condition of instability of the trivial solution (9). The fulfillment of the condition (11) ensures the realization of the resonant repeller (9). Let us assume that $\left\{\omega_{x}>0, \omega>0\right\}$. In this case, asymptotic analysis of conditions (10), (11) makes it possible to distinguish the following twelve typical cases of resonant attractor or resonant repeller realization: 1) if $\left\langle d \omega_{x} / d t\right\rangle<0, d \omega / d t<0, \omega_{x}(0)>\max \omega_{x}^{r}>0$, condition (10) is fulfilled and resonant attractor (9) is realized; 2) if $\left\langle d \omega_{x} / d t\right\rangle>0, d \omega / d t>0, \max \omega_{x}^{r}>\omega_{x}(0)>\omega_{x}^{r}(0)>0$, condition (11) is fulfilled and resonant repeller (9) is realized; 3) if $\left\langle d \omega_{x} / d t\right\rangle>0, d \omega / d t<0, \omega_{x}\left\langle\frac{d \omega_{x}}{d t}\right\rangle<-\omega \frac{d \omega}{d t}, \omega_{x}^{r}(0)>\omega_{x}(0)>0$, condition (10) is fulfilled and attractor (9) is realized; 4) if $\left\langle d \omega_{x} / d t\right\rangle>0, d \omega / d t<0, \omega_{x}\left\langle\frac{d \omega_{x}}{d t}\right\rangle>-\omega \frac{d \omega}{d t}, \omega_{x}^{r}(0)>\omega_{x}(0)>0$, condition (11) is fulfilled and repeller (9) is realized; 5) if $\left\langle d \omega_{x} / d t\right\rangle<0, d \omega / d t>0, \omega_{x}\left\langle\frac{d \omega_{x}}{d t}\right\rangle<-\omega \frac{d \omega}{d t}, \omega_{x}(0)>\max \omega_{x}^{r}>0$, condition (10) is fulfilled and attractor (9) is realized; 6) if $\left\langle d \omega_{x} / d t\right\rangle<0, d \omega / d t>0,-\omega_{x}\left\langle\frac{d \omega_{x}}{d t}\right\rangle<\omega \frac{d \omega}{d t}, \omega_{x}^{r}(0)>\omega_{x}(0)>0$, condition (11) is fulfilled and repeller (9) is realized; 7) if $\left\langle d \omega_{x} / d t\right\rangle<0, d \omega / d t>0, \omega_{x}\left\langle\frac{d \omega_{x}}{d t}\right\rangle>-\omega \frac{d \omega}{d t}, \omega_{x}(0)>\max \omega_{x}^{r}>0$, condition (10) is fulfilled and repeller (9) is realized; 8) if $\left\langle d \omega_{x} / d t\right\rangle>0, d \omega / d t>0,0<\omega_{x}(0)<\omega_{x}^{r}(0)$, condition (11) is fulfilled and resonant repeller (9) is realized; 9) if $\left\langle d \omega_{x} / d t\right\rangle>0, d \omega / d t<0, \omega_{x}\left\langle\frac{d \omega_{x}}{d t}\right\rangle>-\omega \frac{d \omega}{d t}, \omega_{x}(0)>\max \omega_{x}^{r}>0$, condition (11) is fulfilled and repeller (9) is realized; 10) if $\left\langle d \omega_{x} / d t\right\rangle<0, d \omega / d t<0,0<\omega_{x}(0)>\omega_{x}^{r}(0)$, the condition (10) is fulfilled and the resonant attractor (9) is realized; 11) if $\left\langle d \omega_{x} / d t\right\rangle<0, d \omega / d t>0,-\omega_{x}\left\langle\frac{d \omega_{x}}{d t}\right\rangle>\omega \frac{d \omega}{d t}, \omega_{x}^{r}(0)>\omega_{x}(0)>0$, condition (11) is fulfilled 
and repeller (9) is realized; 12) if $\left\langle d \omega_{x} / d t\right\rangle>0, d \omega / d t<0, \omega_{x}\left\langle\frac{d \omega_{x}}{d t}\right\rangle<-\omega \frac{d \omega}{d t}, \omega_{x}(0)>\max \omega_{x}^{r}>0$, condition (11) is fulfilled and attractor (9) is realized.

Similarly we consider the case $\left\{\omega_{x}<0, \omega<0\right\}$. In this case, the resonant ratio $\Delta=\left(1-\frac{\bar{I}_{x}}{2}\right) \omega_{x}-\omega_{a}$ at $\bar{I}_{x}=2$ is

$$
\Delta=\sqrt{\omega_{x}^{2}+\omega^{2}} .
$$

In this case, the Lyapunov function is (8). Similar typical twelve cases are following: 13) if $\left\langle d \omega_{x} / d t\right\rangle>0, d \omega / d t>0$, $\omega_{x}(0)<\min \omega_{x}^{r}<0$, condition (10) is fulfilled and resonant attractor (9) is realized; 14) if $\left\langle d \omega_{x} / d t\right\rangle<0$, $d \omega / d t<0, \min \omega_{x}^{r}<\omega_{x}(0)<\omega_{x}^{r}(0)<0$, condition (10) is fulfilled and repeller (9) is realized; 15) if $\left\langle d \omega_{x} / d t\right\rangle<0$, $d \omega / d t>0, \omega_{x}\left\langle\frac{d \omega_{x}}{d t}\right\rangle<-\omega \frac{d \omega}{d t}, 0>\omega_{x}(0)>\omega_{x}^{r}(0)$, condition (10) is fulfilled and attractor (9) is realized); 16) if $\left\langle d \omega_{x} / d t\right\rangle<0, d \omega / d t>0, \omega_{x}\left\langle\frac{d \omega_{x}}{d t}\right\rangle>-\omega \frac{d \omega}{d t}, 0>\omega_{x}(0)>\omega_{x}^{r}(0)$, condition (11) is fulfilled and repeller (9) is realized; 17) if $\left\langle d \omega_{x} / d t\right\rangle>0, d \omega / d t<0, \omega_{x}\left\langle\frac{d \omega_{x}}{d t}\right\rangle<-\omega \frac{d \omega}{d t}, \omega_{x}(0)<\omega_{x}^{r}(0)<0$, condition (10) is fulfilled and attractor (9) is realized; 18) if $\left\langle d \omega_{x} / d t\right\rangle>0, d \omega / d t<0,0>\omega_{x}(0)>\omega_{x}^{r}(0), \omega_{x}\left\langle\frac{d \omega_{x}}{d t}\right\rangle>-\omega \frac{d \omega}{d t}$, condition (11) is fulfilled and resonant repeller (9) is realized; 19) if $\left\langle d \omega_{x} / d t\right\rangle>0, d \omega / d t<0, \omega_{x}\left\langle\frac{d \omega_{x}}{d t}\right\rangle>-\omega \frac{d \omega}{d t}, \omega_{x}(0)<\omega_{x}^{r}(0)<0$, condition (10) is fulfilled and repeller (9) is realized;20) if $\left\langle d \omega_{x} / d t\right\rangle<0, d \omega / d t<0,0>\omega_{x}(0)>\omega_{x}^{r}(0)$, condition (11) is fulfilled and resonant repeller (9) is realized; 21) if $\left\langle d \omega_{x} / d t\right\rangle<0, d \omega / d t>0, \omega_{x}\left\langle\frac{d \omega_{x}}{d t}\right\rangle>-\omega \frac{d \omega}{d t}, \omega_{x}(0)<\min \omega_{x}^{r}<0$, condition (11) is fulfilled and repeller (9) is realized; 22) if $\left\langle d \omega_{x} / d t\right\rangle>0, d \omega / d t>0,0>\omega_{x}(0)>\omega_{x}^{r}(0)$, condition (10) is fulfilled and resonant attractor (9) is realized; 23) if $\left\langle d \omega_{x} / d t\right\rangle>0, d \omega / d t<0,-\omega_{x}\left\langle\frac{d \omega_{x}}{d t}\right\rangle<\omega \frac{d \omega}{d t}, \omega_{x}^{r}(0)<\omega_{x}(0)<0$, condition (11) is fulfilled and repeller (9) is realized; 24) if $\left\langle d \omega_{x} / d t\right\rangle<0, d \omega / d t>0, \omega_{x}\left\langle\frac{d \omega_{x}}{d t}\right\rangle<-\omega \frac{d \omega}{d t}, \omega_{x}(0)<\min \omega_{x}^{r}<0$, condition (11) is fulfilled and attractor (9) is realized.

\section{Numerical results}

Numerical results obtained from solve of the equations (1)-(4) confirmed fulfillment of the twenty-four cases discussed above. In particular, Fig. 1 shows the dependence of the Lyapunov function on slow variables $\omega_{x}$ and $\omega$ when realization of resonant attractor. This numerical result corresponds to a typical case 5). Fig. 2 shows the dependence of the Lyapunov function on slow variables $\omega_{x}$ and $\omega$ when realization of resonant repeller. The numerical result shown in Fig. 2 corresponds to case 2). The following parameters of the spacecraft and initial conditions of motion were used in the construction of Figs. 1-2: $m=70 \mathrm{~kg}$; $S=0.1 \mathrm{~m}^{2}, L=0.54 \mathrm{~m}, \Delta \bar{m}=0.02, \bar{m}^{A}=0.05, \theta_{1}-\theta_{3}=\pi, I=1 \mathrm{kgm}^{2}, I_{\mathrm{x}}=0.3 \mathrm{kgm}^{2}, V(0)$ is the initial value of the spacecraft velocity, $V(0)=3400 \mathrm{~m} / \mathrm{s}, \vartheta(0)$ is the initial value of the local flight-pass inclination angle, $\vartheta(0)=-0.087 \mathrm{rad}, H(0)$ is the initial value of spacecraft altitude, $H(0)=100 \mathrm{~km}, \varphi(0)=0, \alpha(0)=0.05 \mathrm{rad}, \omega_{x}=10 \mathrm{~s}^{-1}$ (Fig.1); $\Delta \bar{m}=0.005, \bar{m}^{A}=0.05, \theta_{1}-\theta_{3}=0$, $\omega_{x}=15 s^{-1}$ (Fig. 2). Direction of non-resonant evolution of the corresponding variables is indicated in Figs. 1-2 by arrows.

\section{Conclusion and results}

Thus, the use of the method of averaging and Lyapunov's second method made it possible to carry out an asymptotic analysis of the non-resonant evolution of slow variables during the atmospheric descent of the spacecraft with small aerodynamicinertial asymmetry. By doing so, we obtained conditions for realization of the resonant attractor and resonant repeller at arbitrary angles of attack. In addition, we identified ten typical cases of resonant attractor realization and fourteen typical cases of resonant repeller realization. The approximate analytical results of the study correspond to the results of the numerical simulation. The conditions presented in this study indicate that the resonant attractor can become the resonant repeller. It is also possible for a reverse transition. These transitions can occur due to the change of sign of the angular velocity $\omega_{x}$. By analyzing of the stability conditions, we assumed that the asymmetry parameters take constant values. It should be noted that the descent of a spacecraft with variable asymmetry into the atmosphere presents of a practical interest. For example, the variable asymmetry in 
the considered dynamical system can lead to a transition from the resonant attractor to the resonant repeller. Research of such transient modes falls beyond the scope of this study and may be detailed in the following papers.

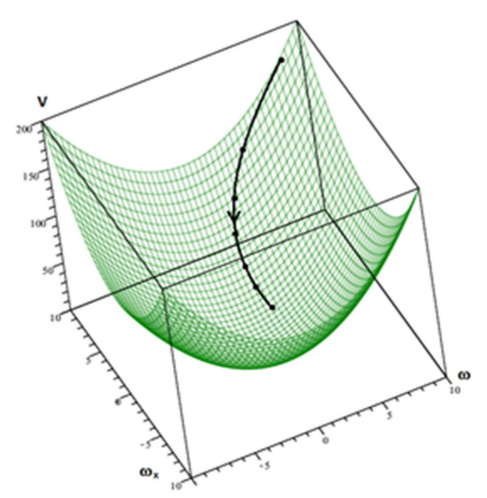

Fig.1. Lyapunov's function and angular velocities when resonant attractor.

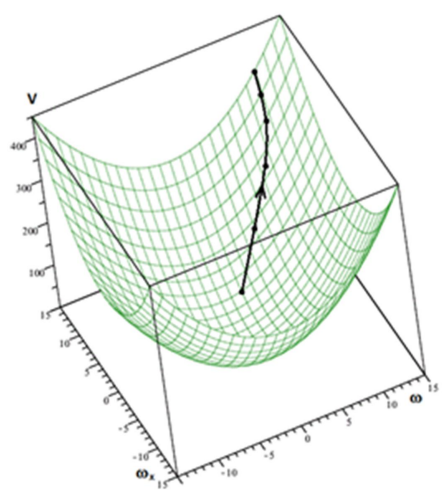

Fig.2. Lyapunov's function and angular velocities when resonant repeller.

\section{References}

[1] Yaroshevskiy VA. Atmospheric reentry of spacecraft. Moscow: Nauka, 1988; 336 p.

[2] Shilov AA, Goman MG. Resonance modes of spatial uncontrolled movement of spacecraft at entry into the atmosphere. Proceedings of TsAGI 1975; 1624: $44 \mathrm{p}$.

[3] Zabolotnov YuM, Lyubimov VV. Secondary resonance effect in the motion of a spacecraft in the atmosphere. Cosmic Research 1998; 36(2): 194-201.

[4] Lyubimov VV. Asymptotic analysis of the secondary resonance effects in the rotation of a spacecraft with a small asymmetry in the atmosphere. Russian Aeronautics 2014; 57(3): 245-252.

[5] Lyubimov VV, Lashin VS. External stability of a resonance during the descent of a spacecraft with a small variable asymmetry in the martian atmosphere. Advances in Space Research 2017; 59(6): 1607-1613.

[6] Sadov YA. Secondary resonance effects in mechanical systems. Mechanics of Solids 1990; 4: 20-24.

[7] Lyubimov VV. External stability of resonances in the motion of an asymmetric rigid body with a strong magnet in the geomagnetic field. Mechanics of Solids 2010; 45(1): 10-21.

[8] Kalaev MP, Lyubimov VV, Semkin ND. Seminatural modeling and numerical simulation for the process of the small satellite separation. Gyroscopy and Navigation 2014; 2(85): 52-60.

[9] Zabolotnov YuM, Lyubimov VV. Application of the method of integral manifolds for construction of resonant curves for the problem of spacecraft entry into the atmosphere. Cosmic Research 2003; 41(5): 453-459.

[10] Shchepakina E, Sobolev V, Mortell MP. Singular perturbations: Introduction to system order reduction methods with applications. Springer Lecture Notes in Mathematics, 2014; 212 p. 